\title{
POINTS OF BOUNDED HEIGHT ON DEL PEZZO SURFACES
}

\author{
Yu. I. Manin ${ }^{1}$, Yu. Tschinkel ${ }^{2}$
}

1) Steklov Mathematical Institute, 42 Vavilova, Moscow 117966, USSR

2)Department of Mathematics, M.I.T., Cambridge(MA) 02139, U.S.A.

\section{INTRODUCTION}

0.1. Heights. In this paper, we prove some results on the asymptotic behaviour of the number of algebraic points of bounded height on del Pezzo (and more general) rational surfaces. The basic (Weil) height on a coordinatized projective space over an algebraic number field $k$ is given by the formula

$$
h_{\mathcal{O}(1), k}\left(x_{0}: \ldots: x_{n}\right)=\prod_{\nu \in M_{k}} \max \left(\left|x_{i}\right|_{\nu}\right)
$$

where the product is taken over all places $\nu$ of $k$, and local norms $\left|x_{i}\right|_{\nu}$ are defined as multipliers of local additive Haar measures on $k_{\nu}$. More generally, we shall consider various Weil heights $h_{L, k, s^{\prime}, s^{\prime \prime}}$ where $L$ is an invertible sheaf on an algebraic variety $V$ defined over $k$, represented as a quotient of two ample sheaves and $s^{\prime}$ (resp. $s^{\prime \prime}$ ) are some families of sections of $\left(L\right.$ resp. $\left.L^{\prime \prime}\right)$ generating these sheaves. We shall usually denote such a height simply $h_{L}$. The chosen normalization (0.1) makes our heights non-invariant with respect to ground field extensions. In compensation, they possess the following "linear growth property". Denote by $N_{\mathbb{P}^{n}}(-K, H)$ the number of points in $\mathbb{P}^{n}(k)$ whose anticanonical height $h_{-K}(x)=h_{\mathcal{O}(1)}(x)^{n+1}$ does not exceed $H$. Put $d=[k: \mathbb{Q}]$. Then

$$
N_{P^{n}}(-K, H)=c H+\left\{\begin{array}{cc}
O\left(H^{1 / 2} \log H\right) & \text { if } \quad d=n=1 \\
O\left(H^{1-1 / d(n+1)}\right) & \text { otherwise. }
\end{array}\right.
$$

This is a restatement of Schanuel's theorem (cf. [Se]). The constant $c$ depends on $k, n$, and the exact normalization of the height.

0.2. Del Pezzo cubic and quartic surfaces. Consider smooth surfaces $V_{5}$ (resp. $V_{6}$ ) over $k$, which are embedded into $\mathbb{P}^{4}$ (resp. $\mathbb{P}^{3}$ ) as a complete intersection of two quadrics (resp. as a cubic). It is well known that over $\bar{k}, V_{5}$ contains 16 lines, whereas $V_{6}$ contains 27 lines. Let us call $V$ split if all lines are defined already over $k$.

One of the results of this paper, having the most direct number-theoretical meaning, can be stated as follows. As above, put $N_{V}(L, H)=\operatorname{card}\left\{x \in V(k) \mid h_{l}(x) \leq\right.$ $H\}$. Notice that $-K_{V_{a}}$ is isomorphic to $\mathcal{O}(1) \mid V_{a}$.

0.3. THEOREM. For a split $V=V_{5}$ and any $\epsilon>0$ we have

$$
N_{V}(O(1), H)=c H^{2}+\left\{\begin{array}{l}
O\left(H^{5 / 4+\epsilon}\right) \\
O\left(H^{3 / 2+\epsilon}\right)
\end{array} \quad \text { if } \quad k=\mathbb{Q},\right.
$$


b) For a split $V=V_{6}$ and any $\epsilon>0$ we have

$$
\begin{array}{ll}
N_{V}(\mathcal{O}(1), H)=c H^{2}+O\left(H^{5 / 3+\epsilon}\right) & \text { if } \quad k=\mathbb{Q}, \\
c H^{2} \leq N_{V}(\mathcal{O}(1), H) \leq c^{\prime} H^{2+\epsilon} & \text { in general. }
\end{array}
$$

Actually, we prove a more precise statement. Namely, the leading term $c H^{2}$ in (0.3), (0.4) counts the number of points of height $\leq H$ on lines whereas the remainder term is an estimate for $N_{U_{a}}(\mathcal{O}(1), H)$, where $U_{a}=V_{a} \backslash\{$ union of lines . This structure of the leading term follows from the Schanuel theorem, and an estimate for $U_{a}$ is our main concern.

0.4. Strategy of proof. More generally, let $V_{a}$ be a split del Pezzo surface over $k$ of degree $d=K_{V}^{2}=9-a$ (cf. e.g. [Ma]). Denote by $U_{a}$ the complement to the union of all exceptional curves (lines) on $V_{a}$. For $a \leq 4$ over $\mathbb{Q}$ (and for $a \leq 3$ in general) we prove directly in section 1 that

$$
N_{U_{a}}(-K, H)=O\left(H^{1+\epsilon}\right) .
$$

The proof uses combinatorial properties of the intersection graph of lines and arithmetics of partial (finite) heights. Then we represent $V_{5}, V_{6}$ as a blow up of $V_{3}$ or $V_{4}$ and apply an estimate of exceptional heights in terms of anticanonical heights, using algebro-geometric arguments.

We know of only one result of this type for cubic surfaces previously discussed in the literature. Namely, C. Hooley $([\mathrm{Ho}])$ proved by a sieve method that the number of points of $x_{0}^{3}+x_{1}^{3}+x_{2}^{3}+x_{3}^{3}=0$ over $\mathbb{Q}$ with height $\leq H$ outside of lines is $O\left(H^{5 / 3+\epsilon}\right)$. However, this surface is not split, so that Hooley's theorem is not contained in ours.

0.5. A perspective. In $[\mathrm{BaMa}]$, we proposed the following general notions related to counting points of bounded height. Let $V$ be a projective variety over a number field $k, L$ an ample sheaf on $V, U$ a quasiprojective subset of $V$. Put

$$
\beta_{U}(L)=\left\{\begin{array}{rr}
\lim \sup \log N_{U}(L, H) / \log H, & \text { if } \\
-\infty & \text { otherwise. }
\end{array}\right.
$$

If $U(k)$ is infinite, there exists a unique minimal Zariski closed subset $Z$ in $U$ such that

$$
\beta_{U}(L)=\beta_{Z}(L)>\beta_{U \backslash Z}(L) .
$$

Of course, $Z$ may coincide with $U$. This subset is called the (minimal) accumulating subset (in $U$ with respect to $L$ ). This notion does not depend on the exact choice of a height $h_{L}$ used to count points.

Repeating this construction, we obtain a sequence of open subsets

$$
V=V_{0} \supset V_{1} \supset \ldots \supset V_{n} \supset \ldots
$$

such that $Z_{i}=V_{i} \backslash V_{i+1}$ is the minimal accumulating subset in $V_{i}$ and a sequence of real numbers $\beta_{i}=\beta_{V_{i}}(L)$. The sequence $\left\{V_{i}\right\}$ is called an arithmetical stratification and $\beta_{i}$ are called growth orders. If $\beta_{i}=1$, we say that $V_{i}$ has a linear growth property (with respect to $L$ ). 
According to the philosophy explained in [BaMa] we expect that if the ground field is made sufficiently large, then both the arithmetical stratification and its growth order should be computable in algebro-geometric terms (although their definitions involve arithmetics). In particular, we expect that for Fano varieties the arithmetical stratification stabilizes at a finite step, and the last growth order with respect to $-K$ equals 1 (again, if the ground field is large enough).

From this viewpoint, what we prove here means in particular that if all exceptional curves on a two-dimensional Fano variety over $\mathbb{Q}$ of degree $d \geq 3$ are defined over $\mathbb{Q}$, they form the first accumulating subset. Moreover, the complement has the linear growth property for $d \geq 5$.

0.6. Plan. The paper is structured as follows. In sec. 1, we prove directly (0.5). In sec. 2, we estimate exceptional heights and deduce the Theorem 0.3. In sec. 3, we discuss the growth orders with respect to other ample sheaves, complementing the results of [BaMa]. Finally, in sec. 4, we derive an approximation to the linear growth conjecture for anticanonical heights on rational surfaces obtained by blowing up rational cycles on a projective plane.

\section{$\S 1$ Finite heights and del Pezzo surfaces of degrees 5 and 6}

1.1 Finite heights. Let $V$ be a projective variety defined over $k, L$ an invertible sheaf on it, $h_{L}$ a Weil height. A choice of an isomorphism $L \simeq \mathcal{O}(D)$ allows one to decompose $h_{L}$ into a product of archimedean and non-archimedean (finite) partial heights: for $x \in V \backslash D, \quad h_{L}(x)=h_{D, \infty} h_{D, f}(x)$. In an Arakelov set up, $h_{D, f}(x)$ is just the product of the exponentiated intersection indices $\langle D, x\rangle_{\nu}$ over archimedean places $\nu \in M_{k, f}$. We do not want to choose a $\mathbf{Z}$-model and hermitean metrics, so that we will rely upon the more elementary version of A.Weil's distributions. In particular, let $D_{i}$ be the divisor $x_{i}=0$ on $\mathbb{P}^{n}$ (see (0.1)). Then

$$
h_{D_{i}, f}\left(x_{0}: \ldots: x_{n}\right)=\prod_{\nu \in M_{k, f}} \max _{j}\left(\left|x_{j} / x_{i}\right|_{\nu}\right)
$$

Our basic technique consists in studying finite heights with respect to lines on the del Pezzo surfaces (it was applied by V.Batyrev in the context of toric varieties). In order to control the resulting loss of information, we start with looking more closely at $\mathbb{P}^{n}$.

Let $A$ be the ring of integers in $K, A^{*}$ the group of units. Choose a family of ideals $a_{1}, \ldots . a_{h} \subset A$ representing all ideal classes, and put

$$
A_{\text {prim }}^{n+1}=\left\{\left(x_{0}: \ldots: x_{n}\right) \in A^{n+1} \mid \exists i, g c d\left(x_{0}, \ldots, x_{n}\right)=a_{i}\right\}
$$

$A^{*}$ acts diagonally upon $A_{\text {prim }}^{n+1}$, and we can identify $\mathbb{P}^{n}(k)$ with $A_{p r i m}^{n+1} / A^{*}$. When we represent a point by its coordinates we usually take coordinates in $A_{\text {prim }}^{n+1}$.

From (1.1) it follows that, for $\left(x_{0}: \ldots: x_{n}\right) \in A_{\text {prim }}^{n+1}, x_{i} \neq 0$ we have

$$
h_{D_{i}, f}=d_{i}(x) N_{k / \mathbb{Q}}\left(x_{i}\right)
$$

where $d_{i}: \mathbb{P}^{n}(k) \rightarrow \mathbb{Q}_{>0}$ is a finite-valued function. In particular, finite heights are "almost integers" for any choice of local Weil's functions (or Arakelov model). 
1.2 Lemma. Let $\eta:\left(\mathbb{P}^{n} \backslash \cup_{i=0}^{n} D_{i}\right)(k) \rightarrow \mathbb{Q}_{>0}^{n+1}$ be the map

$$
\eta(x)=\left(h_{D_{0}, f}(x), \ldots, h_{D_{n}, f}(x)\right) .
$$

Then the number of points $x$ with $h_{\mathcal{O}(1)}(x) \leq H$ having the same image $\eta(x)$ is bounded by $O(1)$, if $k=\mathbb{Q}$, and by $O\left(H^{\epsilon}\right)$ for any $\epsilon>0$, in general.

Proof. For $k=\mathbb{Q},(1.2)$ shows that the knowledge of $h_{D_{i}, f}(x)$ allows us to reconstruct projective coordinates of $x$ up to a finite bounded ambiguity.

In general, knowing the norm $N_{k / \mathbb{Q}}\left(x_{i}\right)$, we can reconstruct first the ideal of $x_{i}$ in $A$ in no more than $O\left(H^{\epsilon}\right)$ ways. In fact, $\left(x_{i}\right)$ divides $\left(N_{k / \mathbb{Q}}\left(x_{i}\right)\right)$, and the number of ideals dividing $\left(N_{k / \mathbb{Q}}\left(x_{i}\right)\right)$ is bounded by $C(\epsilon)\left(N_{k / \mathbb{Q}}\left(x_{i}\right)\right)^{\epsilon}$ (look at the Dedekind zeta of $k$ ).

Fix now a family of ideals $\left(x_{i}\right)$ corresponding to a given $\eta(x)$. From (1.2) one sees that a set of such points is a union of a bounded number of subsets $\left\{\left(x_{0}\right.\right.$ : $\left.\left.\epsilon_{1} x_{1}: \epsilon_{2} x_{2}: \ldots: \epsilon_{n} x_{n}\right)\right\}$ where $\left(x_{0}: \ldots: x_{n}\right)$ are fixed, and $\epsilon_{i} \in A^{*}$ are variable $\left(\epsilon_{0}\right.$ can be killed by the overall multiplication by $A^{*}$ ). Now,

$$
h_{\mathcal{O}(1)}\left(x_{0}: \epsilon_{1} x_{1}: \ldots: \epsilon_{n} x_{n}\right)=\prod_{\nu \in M_{\infty}} \max \left(1,\left|\epsilon_{i} x_{1} / x_{0}\right|_{\nu} \mid i \geq 1\right) h_{D_{0}, f}\left(x_{0}: \ldots: x_{n}\right) .
$$

The left hand side is bounded by $H$ only if

$$
c_{1} H^{-2(n-1)} \leq\left|\epsilon_{i}\right|_{\nu} \leq c_{2} H^{2}
$$

for all $i=1, \ldots, n ; \nu \in M_{\infty}$, and certain $c_{1}, c_{2}>0$. From the Dirichlet theorem it follows that there are no more than $O\left((\log H)^{r}\right)$ units with this property.

Notice that lemma 1.2 is still true if finite heights (1.2) are replaced by equivalent ones.

1.3. Finite exceptional heights on del Pezzo surfaces. Let now $V_{a}$ be a split del Pezzo surface of degree $9-a$ over $k, E_{a}$ the set of exceptional curves (lines) on $V_{a}, U_{a}=V_{a} \backslash \cup_{l \in E_{a}} l$. Choose a family of finite exceptional heighs $h_{l, f}, l \in E_{a}$. We may and will assume that they take values in $\mathbf{Z}_{>0}$. Put now

$$
\tilde{\eta}(x)=\left(h_{l, f}(x)\right) \in \mathbf{Z}_{>0}^{E_{a}}, \quad x \in U_{a}(k)
$$

Generally, we compute $h_{l, f}(x)$ as follows. We represent $l$ as an infimum (or gcd) of divisors $D_{i}$ for which $h_{D_{i}, f}(x)$ are known (e.g. by (1.2)), and then $h_{l, f}(x)=$ $\operatorname{gcd}\left(h_{D_{i}, f}(x)\right)$.

1.4. Lemma. If $a \geq 3$ then the number of points $x \in U_{a}(k)$ with $h_{-K}(x) \leq H$ having the same image $\eta(x)$ doesn't exceed $O(1)$ for $k=\mathbb{Q}$, and $O\left(H^{\epsilon}\right)$ for any $\epsilon>0$.

Proof. Consider a birational morphism $\pi: V_{a} \rightarrow \mathbb{P}^{2}$ blowing down pairwise disjoint lines on $V_{a}$. Choose three lines $D_{1}, D_{2}, D_{3}$ on $\mathbb{P}^{2}$ joining pairwise three fundamental points of $\pi^{-1}$ on $\mathbb{P}^{2}$. For $\{i, j, k\}=\{1,2,3\}$, let $l_{i}=\pi^{-1}\left(D_{j} \cap D_{k}\right)$,, and $l_{i}^{\prime},=\pi^{-1}\left(D_{i}\right)$ (proper inverse image). 
Then, by functorality,

$$
h_{D_{i}, f}(\pi(x))=h_{l_{i}^{\prime}, f}(x) h_{l_{j}, f}(x) h_{l_{k}, f}(x) d_{i}^{\prime}(x)
$$

where $d_{i}^{\prime}$ are finite-valued functions. Hence, knowing $\tilde{\eta}(x)$, we can reconstruct $\eta(\pi(x))$ with a bounded indeterminacy, and then $\pi(x)$ with indeterminacy $O(1)$ for $k=\mathbb{Q}$, and $O\left(H^{\epsilon}\right)$ for any $\epsilon>0$ generally. in view of Lemma 1.2., where $\tilde{H}$ is a bound for $h_{\mathcal{O}(1)}(\pi(x))$. But in view of Lemma 2.2 below, one can take for $\tilde{H}$ a fixed positive power of $H$.

1.5. Remark. We shall consider the family of finite heights

$$
h_{l_{i}, f}(x):=l_{i}(x)
$$

as a marking of the vertices of the intersection graph of $E_{a}$ by positive integers. The proof of Lemma 1.4. shows that (on the set of points $x \in U_{a}(k)$ with $\left.h_{-K}(x) \leq H\right)$ ) the total marking can be reconstructed with indeterminacy $O(1)$, resp. $O\left(H^{\epsilon}\right)$, from the marking of any complete subgraph of the form

Fig.1

This means that for $a \geq 4,\left\{l_{i}(x)\right\}$ must satisfy a system of strong constraints. Some of them are made explicit in the following Lemma.

1.6. Lemma. a). If $l \cap l^{\prime}=\emptyset$, then $\operatorname{gcd}\left(l(x), l^{\prime}(x)\right)$ is a finite valued function ( we shall express this by saying that $l(x)$ and $l^{\prime}(x)$ are almost relatively prime).

b). Consider a complete subgraph $\Delta$ of $E_{a}$ of the form

Then there exist functions

Fig. 2

$$
\left(\sigma_{1}, \sigma_{2}, \sigma_{3}\right): U_{a}(k) \rightarrow A_{p r i m}^{3}
$$

and finite-valued functions $d_{i}: U_{a}(k) \rightarrow \mathbb{Q}^{*}, i=1,2,3$, such that

$$
\begin{gathered}
\sigma_{1}(x)+\sigma_{2}(x)+\sigma_{3}(x)=0, \\
N_{k / \mathbb{Q}}\left(\sigma_{i}(x)\right)=d_{i}(x) l_{i}(x) l_{i}^{\prime}(x) .
\end{gathered}
$$

Proof. The first statement is classical, it is due to A.Weil. In order to prove the second statement, we first notice that any complete subgraph of type $\Delta$ consists of three degenerate fibers of a morphism $\rho: V_{a} \rightarrow P^{1}$ representing $V_{a}$ as a conic bundle. Hence we can find three sections $s_{1}, s_{2}, s_{3}$ of $\rho^{*}(\mathcal{O}(1))$ such that $s_{1}+s_{2}+$ $s_{3}=0$ and $l_{i} \cup l_{i}^{\prime}=\left\{s_{i}=0\right\}$ Primitive coordinates of $\rho(x)$ with respect to these three sections define functions $\sigma_{i}(x)$. Formula (1.3) then follows from (1.2).

We can now prove two main results of this section. 
1.7. Theorem. For $V=V_{3}$ over an arbitrary number field $k$ we have:

$$
N_{U_{3}}(-K, H)=\left\{\begin{array}{ccc}
O\left(H(\log H)^{5}\right) & \text { for } & k=\mathbb{Q}, \\
O\left(H^{1+\epsilon}\right) & \text { for } \quad \text { any } \epsilon>0 & \text { in general } .
\end{array}\right.
$$

Proof. The intersection graph of $E_{3}$ is a hexagon (Fig. 1), and $-K_{V_{3}}=\sum_{i=1}^{3}\left(l_{i}+\right.$ $\left.l_{i}^{\prime}\right)$. Therefore, for $x \in U_{3}(k)$,

$$
h_{-K}(x)=\exp (O(1)) \prod_{i=1}^{3} h_{l_{i}+l_{i}^{\prime}}(x) \geq B \prod_{i=1}^{3} l_{i}(x) l_{i}^{\prime}(x) .
$$

Considering $\left\{l_{i}(x), l_{i}^{\prime}(x)\right\}$ as independent integer variables, we see that points $x$ with $h_{-K}(x) \leq H$ define $O\left(H(\log H)^{5}\right)$ markings of $E_{3}$, whereas every marking corresponds to $O(1)$ ( resp. $O\left(H^{\epsilon}\right)$ ) points.

1.8. Remark. For $k=\mathbb{Q}$, in $[\mathrm{BaMa}]$ it was proved that

$$
N_{U_{3}}(-K, H)=\exp (O(1)) H(\log H)^{3} .
$$

It would be important to know the correct power of logarithm in general.

Furthermore, using the morphism $\pi: V_{3} \rightarrow \mathbb{P}^{2}$, one sees that $N_{U_{3}}(-K, H) \geq$ $\exp (O(1)) H$, so that $\beta_{U_{3}}(-K)=1$.

1.9. Theorem. For $V=V_{4}$ over $k=\mathbb{Q}$ and arbitrary $\epsilon>0$, we have

$$
N_{U_{4}}(-K, H)=O\left(H(\log H)^{6}\right) .
$$

Proof. We start with choosing finite heights $h_{l, f}: U_{4}(\mathbb{Q}) \rightarrow \mathbf{Z}_{>0}, l \in E_{4}$. Represent $U_{4}(\mathbb{Q})$ as a union of subsets $U^{(l)}$ such that

$$
x \in U^{(l)} \Rightarrow l(x)=\min _{l^{\prime} \in E_{4}}\left\{l^{\prime}(x)\right\} .
$$

Clearly, it suffices to prove the estimate $O\left(H(\log H)^{6}\right)$ for points in every subset $U^{(l)}$. Fix $l=l_{0}$ and consider a complete subgraph $\Gamma$ of $E_{4}$ of the form 
Since the automorphism group of the intersection graph of $E_{a}, a \geq 3$, is transitive on vertices, it suffices to exhibit one complete subgraph isomorphic to $\Gamma$. Representing $V_{4}$ by $\mathbb{P}^{2}$ blown up at points $p_{0}, \ldots, p_{3}$, and denoting their preimages $\lambda_{i}$, and inverse image of the line joining $p_{i}, p_{j}$ by $\lambda_{i j}$, we can describe $\Gamma$ by the following identifications:

$$
\begin{array}{cccccccc}
l_{o} & l_{1} & l_{2} & l_{3} & l_{4} & l_{5} & l_{6} & l_{7} \\
\lambda_{0} & \lambda_{01} & \lambda_{1} & \lambda_{12} & \lambda_{2} & \lambda_{23} & \lambda_{3} & \lambda_{13}
\end{array}
$$

Table 1

Using the same model, one sees that $-K=\sum_{i=1}^{5} l_{i}$. Hence we can weaken the inequality $h_{K}(x) \leq H$ to $\prod_{i=1}^{5} l_{i}(x) \leq H$, which has $O\left(H(\log H)^{4}\right)$ solutions. However, we cannot prove that one can reconstruct $x$ even with indeterminacy $O\left(H^{\epsilon}\right)$ from $l_{1}(x), \ldots l_{5}(x)$. Therefore we shall apply the following trick. Put $l_{2}^{\prime}(x)=$ $\left[l_{2}(x) / l_{0}(x)\right] \geq 1$ and weaken the inequality $h_{-K}(x) \leq H$ to

$$
l_{0}(x) l_{1}(x) l_{2}^{\prime}(x) l_{3}(x) l_{4}(x) l_{5}(x) \leq H
$$

Consider the members in the l.h.s. of (1.4) as independent variables. The number of solutions of (1.4) is $O\left(H\left(\log (H)^{5}\right)\right.$, but now we shall be able to reconstruct $x$ with desired ambiguity. We shall first explain how to get $O\left(H^{\epsilon}\right)$.

i) Reconstruction of $l_{6}(x), l_{7}(x)$. Consider the subgraph of $\Gamma:\left(l_{0}, l_{1}, l_{3}, l_{4}, l_{6}, l_{7}\right)$. We can apply to it Lemma 1.4 and obtain the relations (here the assumption $k=\mathbb{Q}$ is used):

$$
d_{1}(x) l_{0}(x) l_{1}(x)+d_{2}(x) l_{3}(x) l_{4}(x)+d_{3}(x) l_{6}(x) l_{7}(x)=0
$$

Knowing $l_{0}, l_{1}, l_{3}, l_{4}$, we can reconstruct $l_{6}, l_{7}$ in $O(\tau(a))$ ways where $\tau$ is the number of divisors, and $a=O(H)$. Clearly, $\max \{\tau(a) \mid a \leq H\}=O\left(H^{\epsilon}\right)$ for any $\epsilon>0$.

ii) Reconstruction of $l_{2}(x) \bmod l_{0}(x)$. To do this, consider a different complete subgraph of $E_{a}$, isomorphic to the one of Fig.2. It is not contained in $\Gamma$. In terms of the description given at the beginning of the proof it can be identified as $\left(l_{2}, l_{7} ; l_{4}, l_{5} ; l_{0}, \lambda_{03}\right)$. From the relations

$$
d_{1}^{\prime}(x) l_{2}(x) l_{7}(x)+d_{2}^{\prime}(x) l_{4}(x) l_{5}(x)+d_{3}^{\prime}(x) l_{0}(x) \lambda_{03}(x)=0
$$

It follows that knowing $l_{7}(x), l_{4}(x), l_{5}(x)$ one can reconstruct $l_{2}(x) \bmod l_{0}(x)$ up to a finite ambiguity. Here we use the fact that $l_{7}(x)$ and $l_{0}(x)$ are almost relatively prime (Lemma 1.6 a)).

iii) Reconstruction of $x$. We can now reconstruct the finite height $l_{2}(x)$, because we know $\left[l_{2}(x) / l_{0}(x)\right]$ and $l_{2}(x) \bmod l_{0}(x)$. And, since we know already the marking of a hexagon, we can reconstruct $x$ up to a finite ambiguity.

Now we will prove a sharper estimate replacing $H^{\epsilon}$ by $(\log H)^{6}$. We are thankful to Don Zagier for help. We start with a refinement of relations (1.3).

Split $V_{4}$ has no moduli, and we can normalise $l_{i}(x)$ for $x \in U_{4}(\mathbb{Q})$ in such a way that quadratic relations of Lemma 1.6 take a canonical form. Choose coordinates in $\mathbb{P}^{2}$ in such a way that $\pi: V_{4} \rightarrow \mathbb{P}^{2}$ blows up points $P_{1}=(1: 0: 0), P_{2}=(0$ : $1: 0), P_{3}=(0: 0: 1), P_{4}=(1: 1: 1)$. Let $x \in U_{3}(\mathbb{Q})$. Then $\pi(x)$ can be represented by $\left(x_{1}, x_{2}, x_{3}\right) \in \mathbf{Z}_{\text {prim }}^{3}$. Define the following ten integers: $d_{i}=\operatorname{gcd}\left(x_{j}, x_{k}\right)$, 
$y_{i}=x_{i} / d_{j} d_{k},\{i, j, k\}=\{1,2,3\} ; D=\operatorname{gcd}_{i \neq k}\left\{y_{i} d_{k}-y_{k} d_{i}\right\} ; z_{j}=\left|y_{i} d_{k}-y_{k} d_{i}\right| / D$. One can check that they define the marking of $E_{4}$ by the respective finite heights. More precisely, with the notation of the Table 1 , one has the following correspondence:

$$
\begin{array}{ccccccccccc}
l: & \lambda_{0} & \lambda_{01} & \lambda_{1} & \lambda_{12} & \lambda_{2} & \lambda_{23} & \lambda_{3} & \lambda_{13} & \lambda_{02} & \lambda_{03} \\
l(x): & D & z_{1} & \left|y_{1}\right| & d_{3} & \left|y_{2}\right| & d_{1} & \left|y_{3}\right| & d_{2} & z_{2} & z_{3}
\end{array}
$$

The symmetries of this marking are not at all obvious from the direct construction.

Here is the list of all marked subgraphs of the type $\Delta$ (Lemma 1.6):

One can directly check that every quadratic relation corresponding to such a subgraph can be written in the form: maximal product of two marks connected by an edge equals to the sum of two remaining products.

We can call all markings of $E_{4}$, obtained in this way, standard. In the proof of Theorem 1.9 we use a majoration of $h_{-K}(x)$ in terms of the respective marking, but actually we can reconstruct the total anticanonical height:

$$
h_{-K}(x)=\exp (O(1)) \max \left\{\prod_{i=1}^{5} l_{i}(x) \mid l_{i}=\text { vertices of a pentagon }\right\}
$$

We leave (1.5) as an exercise to the reader, since we will not use it. We will make further estimates assuming that the marking $\left\{l_{i}(x)\right\}$ is standard.

Returning to the reconstruction procedure i)-iii) and writing for brevity $l_{i}$ instead of former $l_{i}(x)$ etc. we see that

$$
N_{U^{(l)}}(-K ; H) \leq \text { const. } \sum_{l_{0} l_{1} l_{2}^{\prime} l_{3} l_{4} l_{5} \leq H} \tau\left(\delta_{1} l_{0} l_{1}+\delta_{2} l_{3} l_{4}\right)
$$

where $\delta_{i}=1$ or -1 because our marking is standard. We first estimate the r.h.s. by

$$
\begin{gathered}
\sum_{l_{0} l_{1} l_{3} l_{4} \leq H} \tau\left(\delta_{1} l_{0} l_{1}+\delta_{2} l_{3} l_{4}\right) \sum_{l_{2}^{\prime} l_{5} \leq \frac{l^{\prime}}{l_{0} l_{1} l_{3} l_{4}}} 1= \\
\sum_{l_{0} l_{1} l_{3} l_{4} \leq H} \tau\left(\delta_{1} l_{0} l_{1}+\delta_{2} l_{3} l_{4}\right) \frac{H}{l_{0} l_{1} l_{3} l_{4}}(\log H+O(H))= \\
\left(\sum_{a b \leq H} \frac{\tau(a) \tau(b) \tau\left(\delta_{1} a+\delta_{2} b\right)}{a b}\right)(H \log H+O(1))
\end{gathered}
$$

It remains to show that the r.h.s. sum in $(1.7)$ is $O\left((\log H)^{5}\right)$. 
Step 1. We prove that

$$
g(n):=\sum_{a b \leq n} \tau(a) \tau(b) \tau\left(\delta_{1} a+\delta_{2} b\right)=O\left(n(\log n)^{4}\right)
$$

Notice that $\delta_{i}$ can depend on $a, b$; however, it suffices to prove this estimate for constant $\delta_{i}^{\prime}$ s in two separate cases:

i) when $\delta_{1}=1, \delta_{2}=-1 ; a>b$;

ii) when $\delta_{1}=\delta_{2}=1$.

The second case reduces to the first one if one denotes $a+b$ by $a^{\prime}, b$ by $b^{\prime}$, and observes that $a^{\prime} b^{\prime} \leq 2 n$. Now

$$
\sum_{\substack{a b \leq n \\ b<a}} \tau(a) \tau(b) \tau(a-b)=\sum_{b \leq n} \tau(b) \sum_{b<a \leq \frac{n}{b}} \tau(a) \tau(a-b) ;
$$

For a fixed $b$

$$
\begin{gathered}
\sum_{b<a \leq \frac{n}{b}} \tau(a) \tau(a-b) \leq 4 \sum_{\substack{p \geq q>0, r \geq s>0 \\
\frac{n}{b} \geq p q=r s+b}} 1 \leq 4 \sum_{\substack { 0<q, s \leq \sqrt{\frac{n}{b}} \\
\begin{subarray}{c}{0<p<\frac{n}{b q} \\
p q \equiv b \text { mods }{ 0 < q , s \leq \sqrt { \frac { n } { b } } \\
\begin{subarray} { c } { 0 < p < \frac { n } { b q } \\
p q \equiv b \text { mods } } }\end{subarray}} 1 \\
\leq 4 \sum_{\substack{0, s \leq \sqrt{\frac{n}{b}} \\
0<q, s}} \omega(q, s)\left(\frac{n}{b q s}+1\right)
\end{gathered}
$$

where

$$
\omega(q, s)=\operatorname{card}\{p(\bmod s) \mid p q \equiv b(\bmod s)\}
$$

equals $d:=(q, s)$ if $d$ divides $b$, and 0 otherwise. We continue to estimate (1.9):

$$
\begin{aligned}
& \quad \leq 4 \sum_{d \mid b} d \sum_{\substack{0<q, s \leq \sqrt{\frac{n}{b}} \\
(q, s)=0(d)}}\left(\frac{n}{b q s}+1\right) \leq \\
& \leq 4 \sum_{d \mid b} d \sum_{\substack{0<x, y \leq \frac{1}{d} \sqrt{\frac{n}{b}}\\
}}\left(\frac{n}{b d^{2} x y}+1\right) \\
& \leq 4 \sigma_{-1}(b)\left(\frac{n}{b} \log ^{2} \frac{n}{b}+O\left(\frac{n}{b} \log \frac{n}{b}\right)\right)
\end{aligned}
$$

where $\sigma_{-1}(b)=\sum_{d \mid b} \frac{1}{d}$. Now (1.8) becomes

$$
\leq 4 \sum_{b \leq n} \tau(b) \sigma_{-1}(b)\left(\frac{n}{b} \log ^{2} \frac{n}{b}+O\left(\frac{n}{b} \log \frac{n}{b}\right)\right)=O\left(n(\log n)^{4}\right) .
$$

Step 2. Now apply Abel's summation:

$$
\sum_{a b \leq H} \frac{\tau(a) \tau(b) \tau\left(\delta_{1} a+\delta_{2} b\right)}{a b}=O\left((\log H)^{5}\right) .
$$




\section{EXCEPTIONAL HEIGHTS}

In this section, we shall prove the following inductive estimate (cf. 0.5 for notation).

2.1. PROPOSITION. Assume that for a given ground field $k$ some $a \geq 2$ and all split del Pezzo surfaces $V_{a}$ of degree $9-a$ over $k$ we have

$$
\beta_{U_{a}}(-K) \leq \beta_{a}
$$

where $U_{a}=V_{a} \backslash\{$ all lines $\}$, and $\beta_{a}$ is a constant. Then the same is true for all split del Pezzo surfaces $V_{a+1}$ with $\beta_{a}$ replaced by $\beta_{a+1}=\frac{9-a}{8-a} \beta_{a}$.

For example, if we know that $\beta_{4}=1$, we deduce that $\beta_{5}=5 / 4$ and $\beta_{6}=5 / 3$; and if we know only that $\beta_{3}=1$, we get $\beta_{4}=6 / 5$, and $\beta_{5}=3 / 2, \beta_{6}=2$. Thus the Theorem 0.3 follows from the Proposition 2.1 and the results of the previous section.

We start with the following auxiliary result.

2.2.LEMMA. Let $a \geq 2$. Denote by $\left\{l_{1}, \ldots, l_{e(a+1)}\right\}$ the set of all exceptional curves of the first kind (lines) on a del Pezzo surface $V=V_{a+1}$ of degree $8-a$. The class of their sum in Pic $(V)$ equals $(e(a+1) /(8-a))\left(-K_{V}\right)$.

PROOF. Consider the formal symmetry group $W_{a+1}$ of the configuration of lines $\left\{l_{i}\right\}$,that is, the group of the permutations of lines, conserving their intersection indices. From the classical identification of this group with a Weyl group, it follows that the subgroup of $W_{a+1}$-invariant elements is cyclic, with generator $-K$. In order to identify the coefficient, it suffices to intersect both sides with $-K$.

2.3. COROLLARY. Choose some Weil heights $h_{-K}$ and $h_{l_{i}}$ for all $i$.Then there exists a constant $A$ such that for every $x \in U_{a+1}(k)$ one can find a line $l=l(x)$ with the property

$$
h_{l}(x) \leq A\left(h_{-K}(x)\right)^{1 /(8-a)}
$$

PROOF. From the Lemma 2.2 and general properties of heights it follows that

$$
\left.\prod_{i=1}^{e(a+1)} h_{l_{i}}(x)=\exp (O(1))\left(h_{-K}(x)\right)\right)^{e(a+1) /(8-a)}
$$

Now (2.1) is obvious.

Notice that the same argument shows the existence of another exceptional height $h_{l^{\prime}}$,for which

$$
h_{l^{\prime}}(x) \geq B\left(h_{-K}(x)\right)^{1 /(8-a)},
$$

so that exceptional heights have infinitely often the same growth order as ample heights.

2.4. Proof of the proposition 2.1. Fix $k$, a split del Pezzo surface $V_{a+1}$, and some heights $h_{K}, h_{l_{i}}$ on $V_{a+1}(k)$. Corollary 2.3 allows us to define a partition of $U(k)$ into a finite number of subsets $U_{l}$ numbered by lines $l$ such that $(2.1)$ is valid in $U_{l}$. It suffices to prove that the number of points of $\left(-K_{a+1}\right)$-height $\leq H$ in $U_{l}$ is $O\left(H^{\beta_{a+1}+\epsilon}\right)$ where $\beta_{a+1}=((9-a) /(8-a)) \beta_{a}, K_{a+1}=K\left(V_{a+1}\right)$. Embed 
$l$ into a maximal system of pairwise disjoint lines on $V_{a+1}: l_{1}, \ldots, l_{a}, l_{a+1}=l$ (This is always possible: cf. [Ma]). Denote by $\pi: V_{a+1} \rightarrow \mathbb{P}^{2}$ the morphism which blows down this system. Let $\Lambda$ be the class of $\pi^{*}(O(1))$ in $\operatorname{Pic}\left(V_{a+1}\right)$ Choosing all necessary Weil heights, we have for $x \in U_{l}$ :

$$
\begin{gathered}
h_{-K_{a+1}}(x)=\exp (O(1)) h_{3 \Lambda-l_{1}-\ldots-l_{a+1}}(x)= \\
\exp (O(1)) h_{l}(x)^{-1} h_{3 \Lambda-l_{1}-\ldots-l_{a}}(x) \geq \\
B h_{-K_{a+1}}(x)^{-1 /(8-a)} h_{-K_{a}}(\sigma(x))
\end{gathered}
$$

where $\sigma: V_{a+1} \rightarrow V_{a}$ blows down $l_{a+1}=l$ and $K_{a}$ is the canonical class of $V_{a}$. Hence for $x \in U_{l}$

$$
h_{-K_{a+1}}(x) \geq C\left(h_{-K_{a}}(\sigma(x))\right)^{(8-a) /(9-a)} .
$$

Finally, by assumption, the number of points $\sigma(x)$ whose $\left(-K_{a}\right)$-height is bounded by $H$, does not exceed $O\left(H^{\beta_{a}+\epsilon}\right)$. This finishes the proof.

\section{§3. GROWTH ORDERS WITH RESPECT TO OTHER AMPLE SHEAVES}

Let $V=V_{r}$ be an arbitrary split del Pezzo surface over a numberfield $k$, represented as a result of blowing up $r \leq 8$ points in $\mathbb{P}^{2}(k), U=U_{r}$ the complement to the union of lines, $L$ an arbitrary ample invertible sheaf on $V_{r}$. Denote by $\alpha(L)$ the (unique) rational number such that $\alpha(L)[L]+K_{V}$ belongs to the boundary of the cone of effective elements of $\operatorname{Pic}(V)$. In [BaMa], the following theorem was proved:

3.1 THEOREM. $\alpha(L) \leq \beta_{U}(L) \leq \alpha(L) \beta_{U}(-K)$.

It follows that for $k=\mathbb{Q}$ and $r \leq 4$, we have $\beta_{U}(L)=\alpha(L)$.

In [BaMa], we have also given an explicit formula for calculation of $\alpha(L)$, in terms of a fixed representation $\pi: V_{r} \rightarrow \mathbb{P}^{2}$ blowing down $r$ lines. Namely, let $\Lambda=\left[\pi^{*}(\mathcal{O}(1))\right]$. Denote by $l_{1}, \ldots l_{r}$ the classes of blown down lines in Pic $(\mathrm{V})$, $L=A \Lambda-B_{1} l_{1}-\ldots-B_{r} l_{r}$.

Consider all classes $E=a \Lambda-b_{1} l_{1}-\ldots-b_{r} l_{r}$ with the following properties: $E$ is either a preimage of a line on $\mathbb{P}^{2}$ under a morphism blowing down a maximal system of $r$ pairwise non-intersecting exceptional curves on $V$, or a preimage of a generator of a quadric under a morphism blowing down a maximal system of $r-1$ pairwise non-intersecting exceptional curves on V. Then

$$
\alpha(L)=\max _{E}\left\{\frac{2+\epsilon}{A a-B_{1} b_{1}-\ldots-B_{r} b_{r}}\right\}, \quad \epsilon=(E . E) .
$$

Clearly, all classes $E$ are contained among the solutions of the following system of diophantine equations:

$$
\begin{aligned}
& a^{2}-b_{1}^{2}-\ldots-b_{r}^{2}=\epsilon, \quad \epsilon=0 \text { or } 1 \\
& 3 a-b_{1}-\ldots-b_{r}=2+\epsilon,
\end{aligned}
$$

for which $a>0$ and $b_{i} \geq 0$. 
We shall give below the complete list of solutions of (3.2) (with $r=8$, and $b$ numbered in decreasing order). The table in [BaMa] contained only solutions with $r \leq 7$, and several solutions with $\epsilon=0$ were missing.

3.2. Solving (3.2). This system for $r=8$ is equivalent to the following one:

$$
\begin{aligned}
& a^{2}-b_{1}^{2}-\ldots-b_{8}^{2}-b_{9}^{2}=-4-4 \epsilon \\
& 3 a-b_{1}-\ldots-b_{8}-b_{9}=0 \\
& b_{9}=2+\epsilon
\end{aligned}
$$

From the first two equations it follows that

$$
\left(a-3 b_{1}\right)^{2}+\left(a-3 b_{2}\right)^{2}+\ldots+\left(a-3 b_{9}\right)^{2}=36(1+\epsilon) .
$$

Hence we can solve (3.2) in three steps:

i). List all $\left(x_{1}^{(i)}, \ldots x_{9}^{(i)}\right)$ for which

$$
x_{1}^{2}+\ldots+x_{9}^{2}=36(1+\epsilon)
$$

and $x_{j} \bmod 3$ do not depend on $j$.

ii).Find a 10-tuple $\left(a^{(i)} ; b_{1}^{(i)}, \ldots, b_{9}^{(i)}\right)$ corresponding to each entry of the list, with $x_{j}^{(i)}=a^{(i)}-3 b_{j}^{(i)}$; leave only those for which $3 a^{(i)}=\sum b_{j}^{(i)}$.

iii).In each progression $\left(a^{(i)}+3 N, b_{1}^{(i)}+N, \ldots, b_{9}^{(i)}+N\right)$ leave only solutions with positive $a$ and non-negative $b$ 's, one of which equals $2+\epsilon$. Delete $b=2+\epsilon$ and renumber $b$ 's in decreasing order.

3.3. All solutions to (3.2) can be used in (3.1). To prove this one can argue as follows.

First, check directly that all classes $E$ corresponding to the solutions of (3.2) are effective. To establish this, it suffices to present every $E$ as a sum of exceptional classes listed in [Ma], Proposition 26.1.

Second, from Riemann-Roch it follows that $\operatorname{dim}|E| \geq 1+\epsilon$ Therefore, every point $l$ of the closure of effective cone satisfies $(E . l) \geq 0$ for every $E$.

Hence, even if among $E$ 's there were some hyperplanes not contained in the set of walls of this cone, they would not contribute into (3.1).

\section{SOLUTIONS OF (3.2) WITH $\epsilon=0$}




$\begin{array}{rrrrrrrrr}a & b_{1} & b_{2} & b_{3} & b_{4} & b_{5} & b_{6} & b_{7} & b_{8} \\ 1 & 1 & 0 & 0 & 0 & 0 & 0 & 0 & 0 \\ 2 & 1 & 1 & 1 & 1 & 0 & 0 & 0 & 0 \\ 3 & 2 & 1 & 1 & 1 & 1 & 1 & 0 & 0 \\ 4 & 2 & 2 & 2 & 1 & 1 & 1 & 1 & 0 \\ 5 & 2 & 2 & 2 & 2 & 2 & 2 & 1 & 0 \\ 4 & 3 & 1 & 1 & 1 & 1 & 1 & 1 & 1 \\ 5 & 3 & 2 & 2 & 2 & 1 & 1 & 1 & 1 \\ 6 & 3 & 3 & 2 & 2 & 2 & 2 & 1 & 1 \\ 7 & 3 & 3 & 3 & 3 & 2 & 2 & 2 & 1 \\ 8 & 3 & 3 & 3 & 3 & 3 & 3 & 3 & 1 \\ 7 & 4 & 3 & 2 & 2 & 2 & 2 & 2 & 2 \\ 8 & 4 & 3 & 3 & 3 & 3 & 2 & 2 & 2 \\ 9 & 4 & 4 & 3 & 3 & 3 & 3 & 3 & 2 \\ 10 & 4 & 4 & 4 & 4 & 3 & 3 & 3 & 3 \\ 11 & 4 & 4 & 4 & 4 & 4 & 4 & 4 & 3\end{array}$


SOLUTIONS OF (3.2) WITH $\epsilon=1$

\begin{tabular}{|c|c|c|c|c|c|c|c|}
\hline$a$ & $b_{1}$ & $b_{2}$ & $b_{3}$ & $b_{4}$ & $b_{5}$ & $b_{6}$ & $b_{7}$ \\
\hline 1 & 0 & 0 & 0 & 0 & 0 & 0 & 0 \\
\hline 2 & 1 & 1 & 1 & 0 & 0 & 0 & 0 \\
\hline 3 & 2 & 1 & 1 & 1 & 1 & 0 & 0 \\
\hline 4 & 2 & 2 & 2 & 1 & 1 & 1 & 0 \\
\hline 5 & 2 & 2 & 2 & 2 & 2 & 2 & 0 \\
\hline 4 & 3 & 1 & 1 & 1 & 1 & 1 & 1 \\
\hline 5 & 3 & 2 & 2 & 2 & 1 & 1 & 1 \\
\hline 5 & 3 & 3 & 1 & 1 & 1 & 1 & 1 \\
\hline 6 & 3 & 3 & 2 & 2 & 2 & 2 & 1 \\
\hline 6 & 3 & 3 & 3 & 2 & 1 & 1 & 1 \\
\hline 7 & 3 & 3 & 3 & 3 & 2 & 2 & 2 \\
\hline 7 & 3 & 3 & 3 & 3 & 3 & 1 & 1 \\
\hline 8 & 3 & 3 & 3 & 3 & 3 & 3 & 3 \\
\hline 6 & 4 & 2 & 2 & 2 & 2 & 1 & 1 \\
\hline 7 & 4 & 3 & 3 & 2 & 2 & 2 & 1 \\
\hline 8 & 4 & 3 & 3 & 3 & 3 & 3 & 1 \\
\hline 8 & 4 & 4 & 3 & 3 & 2 & 2 & 2 \\
\hline 9 & 4 & 4 & 4 & 3 & 3 & 3 & 2 \\
\hline 9 & 4 & 4 & 4 & 4 & 2 & 2 & 2 \\
\hline 10 & 4 & 4 & 4 & 4 & 4 & 3 & 3 \\
\hline 8 & 5 & 3 & 3 & 2 & 2 & 2 & 2 \\
\hline 9 & 5 & 3 & 3 & 3 & 3 & 3 & 3 \\
\hline 9 & 5 & 4 & 3 & 3 & 3 & 2 & 2 \\
\hline 10 & 5 & 4 & 4 & 4 & 3 & 3 & 2 \\
\hline 10 & 5 & 5 & 4 & 4 & 4 & 3 & 3 \\
\hline 11 & 5 & 5 & 4 & 4 & 4 & 3 & 3 \\
\hline 11 & 5 & 5 & 5 & 3 & 3 & 3 & 3 \\
\hline 12 & 5 & 5 & 5 & 4 & 4 & 4 & 4 \\
\hline 12 & 5 & 5 & 5 & 5 & 4 & 3 & 3 \\
\hline 13 & 5 & 5 & 5 & 5 & 5 & 5 & 3 \\
\hline 10 & 6 & 3 & 3 & 3 & 3 & 3 & 3 \\
\hline 11 & 6 & 4 & 4 & 4 & 3 & 3 & 3 \\
\hline 12 & 6 & 5 & 4 & 4 & 4 & 4 & 3 \\
\hline 13 & 6 & 5 & 5 & 5 & 4 & 4 & 4 \\
\hline 14 & 6 & 5 & 5 & 5 & 5 & 5 & 5 \\
\hline 13 & 6 & 6 & 4 & 4 & 4 & 4 & 4 \\
\hline 14 & 6 & 6 & 5 & 5 & 5 & 4 & 4 \\
\hline 15 & 6 & 6 & 6 & 5 & 5 & 5 & 5 \\
\hline 16 & 6 & 6 & 6 & 6 & 6 & 5 & 5 \\
\hline 17 & 6 & 6 & 6 & 6 & 6 & 6 & 6 \\
\hline 15 & 7 & 5 & 5 & 5 & 5 & 5 & 5 \\
\hline
\end{tabular}




\section{§4. AN APPROXIMATION TO THE LINEAR GROWTH CONJEC-} TURE

In this section, we prove the following result. Consider a rational surface $V$ which is obtained from $\mathbf{P}^{2}$ by blowing up a $k$-rational cycle $z, \pi: V \rightarrow \mathbf{P}^{2}$ (z may have infinitely near components).

4.1. THEOREM. For every $\epsilon \geq 0$, there exists an integer $N>0$, an open Zariski dense subset $U \subset V$, a constant $c>0$ and a partition

$$
U(k)=\bigcup_{\alpha=1}^{\infty} U_{\alpha}(k)
$$

with the following properties

a) for all $\alpha$, card $U_{\alpha}(k) \leq N$.

b) for all $\alpha$,

$$
\max _{x \in U_{\alpha}} h_{\mathcal{O}(1)}(\pi(x)) \leq c \min _{x \in U_{\alpha}} h_{\mathcal{O}(1)}(\pi(x))
$$

c) one can choose a point $x_{\alpha}$ in each subset in $U_{\alpha}(k)$ in such a way that the series

$$
\sum_{\alpha} h_{-K_{V}}\left(x_{\alpha}\right)^{-1-\epsilon}
$$

will converge.

Comment.For a del Pezzo surface $V$, we expect ([BaMa]) that $\beta_{U}\left(-K_{V}\right)=1$. This would follow from the Theorem 4.1, if we could take $N=1$. On the other hand, our statement holds even without assumption that $V$ is del Pezzo. Its main interest probably lies in the method of proof, which is one more variation of the general idea that "heights with respect to the exceptional divisors should be small in average".

4.2. PROOF. Consider a large integer $\mathrm{M}$ and $\mathrm{M}$ elements $i d=g_{1}, \ldots, g_{M}$ of $A u t_{k} \mathbf{P}^{2}$ in a sufficiently general position. Put $z_{i}=g_{i}(z)$ where $z$ is the cycle we have blown up to obtain $V$. Denote by $\mathrm{W}$ the result of blowing up $\cup z_{i}$, and by $V$ the result of blowing up $z_{i}$. Obviously, $g_{i}$ induces an isomorphism of $V=V_{1}$ with $V_{i}$. Moreover, there is a canonical birational morphism $f_{i}: W \rightarrow V_{i}$.

In order to shorten notation, we shall consider inverse image maps $f_{i}^{*}$ on Picgroups as embeddings, and $f_{i}$ themselves as identifications outside their fundamental sets. Let $K_{i}$ be the canonical class of $V_{i}$ (in Pic W) and $\Lambda$ the inverse image of $\mathcal{O}_{\mathbf{P}^{2}}(1)$. Put $Z_{i}=3 \Lambda+K_{i}$.

Denote by $P=P(M)$ the smallest integer for which the linear system $\mid P \Lambda-$ $Z_{1}-\ldots-Z_{M} \mid$ is non-empty. From Riemann-Roch, it follows that $P \leq a M^{1 / 2}$ for an appropriate constant $a$. As in the proof of the Corollary 2.3, we obtain that for every point $x \in U(k)$ where $U$ is the complement to the fundamental points and fixed components of $\left|P \Lambda-Z_{1}-\ldots-Z_{M}\right|$ there exists an $i=i(x)$ such that

$$
h_{Z_{i}}(x) \leq C\left(h_{\Lambda}(x)\right)^{a / M^{1 / 2}}
$$

Here the constant $C$ can be taken independent on $i$. 
Put $\eta=a / M^{1 / 2}$; taking large M, we can make $\eta$ arbitrarily small. For a fixed i, let $W_{i}$ be the set of all points of $U(k)$ for which (4.1) holds. In $W_{i}$, we have

$$
h_{-K_{i}}(x)=\exp (O(1)) h_{Z_{i}}(x)^{-1} h_{3 \Lambda}(x) \geq B h_{\Lambda}(x)^{3-\eta} .
$$

From (4.2) and Schanuel's theorem it follows that

$$
\sum_{x \in W_{i}} h_{-K_{i}}(x)^{-1-\epsilon}
$$

converges for some $\epsilon$ tending to zero when $\eta$ tends to zero.

Now, if $x \in W_{1}$ and if the heights are appropriately normalized, then $g_{i}(x) \in$ $W_{i}$. It follows that we can construct the partition with the properties stated in the Theorem by collecting in one subset points $\left\{g_{1}(x), g_{2}(x), \ldots g_{M}(x)\right\}$ and then deleting multiple occurencies.

Acknowledgements. We would like to thank the Department of Mathematics of M.I.T. and the Max-Planck-Institut für Mathematik in Bonn where parts of this work were done for financial support and stimulating atmosphere. We also thank the referee who detected a mistake in the initial version of Lemma 1.2.

\section{REFERENCES}

[BaMa] V.V.Batyrev, Yu.I.Manin. Sur le nombre des points rationnels de hauteur bornée des variétés algébriques. Math. Annalen, Bd. 286 (1990), 27-43.

[Ho] C. Hooley. On the numbers that are representable as the sum of two cubes. Journ. für die reine und angew. Math., Bd. 314 (1979), 147-173.

[Ma] Yu. I. Manin. Cubic surfaces. North-Holland, Amsterdam, 1974, 2nd edition 1986.

[Se] J.-P. Serre. Lectures on the Mordell-Weil theorem. Vieweg. BraunschweigWiesbaden, 1989. 